\title{
ON A THEOREM OF HARTMAN AND WINTNER
}

\author{
P. W. MILLAR
}

(Communicated by Wei Y. Loh)

\begin{abstract}
An elementary stochastic process argument is given for a measuretheoretic result of Hartman-Wintner, which asserts (under a natural condition) that an infinitely divisible measure has no atoms iff its corresponding Lévy measure has infinite mass.
\end{abstract}

\section{INTRODUCTION}

Let $\mu$ be an infinitely divisible measure on the line. The Lévy-Khinchine representation asserts that $\int e^{i u x} \mu(d x)$ has the form

$$
\exp \{\psi(u)\}
$$

where

$$
\psi(u)=i u \gamma-\sigma^{2} u^{2}+\int\left\{e^{i u x}-1-\frac{i u x}{1+x^{2}}\right\} \nu(d x)
$$

with $\gamma \in R^{\prime}, \sigma^{2} \geq 0$, and $\nu$ a sigma finite measure on $R^{\prime}$ such that $\nu(\{0\})=$ $0, \int_{-1}^{1} x^{2} \nu(d x)<\infty, \nu\{x:|x|>1\}<\infty$. The measure $\nu$ is called the Lévy measure.

In a seminal paper, Hartman and Wintner (1942) showed that, assuming $\sigma^{2}=0$,

$$
\mu \text { has no atoms iff } \nu\left(R^{\prime}\right)=+\infty
$$

(it is trivial that $\mu$ has no atoms if $\sigma^{2}>0$ ). Seventeen years later, Blum and Rosenblatt (1959) rediscovered this result. Direct proof of (1.2) using the form of the characteristic function (1.1) appears difficult. Indeed, both proofs mentioned above take an indirect route: it is first shown that an infinitely divisible distribution is of "pure type"; then (1.2) is deduced at the end by eliminating all of the other possible types.

On the other hand, for the study of processes with independent increments ("Lévy processes"), the characterization given in (1.2) turns out to be quite useful, appearing, for example, in the analysis of first exit times from an interval, last exit times from a point, and so forth. Apparently, none of the other characterizations of type in terms of the Lévy measure have had any application in stochastic process theory.

Received by the editors October 15, 1993.

1991 Mathematics Subject Classification. Primary 60E07.

Research supported by NSA Grant MDS 904-89-H-2045 and by NSF Grant DMS 9001710. 
Accordingly, it seems reasonable to give a direct proof of (1.2), accessible to students of stochastic processes. The next section presents such a proof, based on simple, purely sample-function-theoretic ideas. We emphasize that this proof is not necessarily "better" or "more elementary" than the measure-theoretic technique of Hartman-Wintner. Rather, it is just fascinating to see how a good measure-theoretic result can be quickly derived by soft sample function analysis and the drawing of a few pictures.

\section{MAin Result}

The proposition to be proved is:

Proposition. If $\sigma^{2}=0$, then $\mu$ has no atoms if and only if $\nu\left(R^{\prime}\right)=+\infty$.

To prove this, we shall use elementary facts from the theory of Lévy processes, which can be found in Gikhman and Skorokhod (1969) and Breiman (1968), for example.

Let $X_{1}$ be a random variable with distribution $\mu$. Then there exists a Lévy process $\left\{X_{t}, t \geq 0\right\}$ whose characteristic functions are

$$
E e^{i u X_{t}}=\exp \{t \psi(u)\}
$$

This process can be constructed to have right continuous paths with left limits. The role of the Lévy measure $\nu$ can be partially understood in light of the following facts, which are immediate consequences of the construction of $\left\{X_{t}\right\}$ given, for example, in the references just cited:

(2.2) Facts. (a) If $A$ is a set in $R^{\prime}$ lying a positive distance from 0 , then

$$
\begin{aligned}
& t \nu(A) \text { is equal to the expected number of } s, \\
& s \leq t, \text { for which } X_{s}-X_{s-} \in A \text {. }
\end{aligned}
$$

(b) $\left\{X_{t}\right\}$ has, w.p.1, an infinite number of jumps in every time interval iff $\nu\left(R^{\prime}\right)=+\infty$.

Of course, (b) is easily deduced from (a); in turn (a) says that the expected number of "jumps of size $A$ " is $t \nu(A)$.

As a final preliminary, recall that a compound Poisson process is a Lévy process with step function paths. The following elementary result may also be found in the above references.

(2.3) Facts. (a) A Lévy process $Y=\left\{Y_{t}\right\}$ is compound Poisson if and only if $E\left\{\exp i u Y_{t}\right\}=\exp t \psi_{Y}(u)$, where $\psi_{Y}(u)=\int\left[e^{i u z}-1\right] \nu_{Y}(d x)$ and $\nu_{Y}\left(R^{\prime}\right)<\infty$.

(b) If a Lévy process has paths that are flat in any interval, it must be C.P.

Indeed, part (a) is Theorem 1, p. 274 in Gikhman-Skorokhod. As for (b), let $T_{\delta}=\inf \left\{s>0: Y_{s+u}=Y_{s}\right.$ for $\left.0 \leq u \leq \delta\right\}$. Then $T_{\delta}+\delta$ is an optional time and so therefore is $L \equiv \lim _{\delta \downarrow 0}\left[T_{\delta}+\delta\right] . \quad L$ is the "time of the first flat spot" in the paths of $Y$. By hypothesis, $P\{L<\infty\}>0$. By stationarity and the strong Markov property, $Y$ must have step function paths after $L$, and so has step function paths starting from 0 . Basic properties of optimal times (also called "stopping times") can be found, for example, in Blumenthal and Getoor (1968), Ch. I, Sec. 6; the strong Markov property is discussed in Ch. I, Secs. 7,8 . Note that the proof below requires only a much simpler version of $2.3(\mathrm{~b})$ : if the paths are flat in $[0, T)(T>0)$, then the process is C.P. 
We can now proceed with the proof.

Proof of Proposition. Part 1. Assume $\nu\left(R^{\prime}\right)=+\infty$; we must show $\mu$ has no atoms.

Assume on the contrary that $\mu$ has an atom. Let $\left\{X_{t}^{\prime}, t \geq 0\right\}$ be a Lévy process with the same distribution as the process $\left\{X_{t}\right\}$ introduced in (2.1), but independent of $\left\{X_{t}\right\}$. Let

$$
Y_{t}=X_{t}-X_{t}^{\prime}
$$

If $X_{1}$ (i.e., $\mu$ ) has an atom somewhere, then of course $Y_{1}$ has an atom at 0 . Let

$$
\alpha=P\left\{Y_{1}=0\right\}>0 .
$$

By a maximal inequality of Lévy (cf. Loève, 1977, p. 259)

$$
P\left\{\sup _{0 \leq s \leq 1} Y_{s}>0\right\} \leq 2 P\left\{Y_{1}>0\right\}=1-\alpha .
$$

The last equality in (2.6) follows from symmetry considerations:

$$
1=P\left\{Y_{1}>0\right\}+P\left\{Y_{1}<0\right\}+\alpha=2 P\left\{Y_{1}>0\right\}+\alpha .
$$

It follows from (2.6) that, with probability at least $\alpha, Y_{s}$ remains at 0 or below for all $s, 0 \leq s \leq 1$. This implies, of course, that for all arbitrarily small initial time intervals, $Y_{s}$ remains non-positive with positive probability. More formally, let $A_{\delta}=\left\{w: Y_{s}(w) \leq 0, s \leq \delta\right\}$. Then

$$
\lim _{\delta \downarrow 0} P\left\{A_{\delta}\right\} \geq \alpha>0 .
$$

By the Blumenthal zero-one law (Blumenthal, 1957), it follows that

$$
\lim _{\delta \downarrow 0} P\left\{A_{\delta}\right\}=1 .
$$

Let $T=\inf \left\{t>0: X_{t}>0\right\}$. Then $T$ is an optimal time and by (2.8), $P\{T>0\}=1$. Thus, $X_{t} \leq 0$ for $0 \leq t<T$, where $T>0$. That is, upon starting at 0 , every path of the process $\left\{Y_{t}\right\}$ immediately enters $(-\infty, 0]$ for a short but positive initial length of time.

On the other hand, the process $Y$ is symmetrically distributed, so, upon starting at 0 every path also immediately enters $[0,+\infty)$ for a short, but positive, initial length of time.

Taking the last two sentences together, we see that $Y$, upon starting at 0 , must remain at 0 for an initial length of time. The facts $(2.3)$ then show that $Y$ must be compound Poisson. On the other hand $Y$ is just the symmetrization of $X$, so its Lévy measure $\nu_{Y}$ is just

$$
\nu_{Y}(A)=\nu(A)+\nu(-A)
$$

Since $Y$ is C.P., $\nu_{Y}(R)<\infty$, and so $\nu(R)<\infty$. This contradiction completes the proof of Part 1.

Part 2, the converse. Assume $\mu$ has no atoms. Then we must show that $\nu\left(R^{\prime}\right)=+\infty$.

This argument can proceed more or less as in Part 1. Assume on the contrary that the conclusion is false: $\nu\left(R^{\prime}\right)<\infty$. The symmetrization $Y$ of $X$, described in Part 1, is then compound Poisson. Then $Y_{1}$ has an atom at 0 ; 
this could not happen unless $X_{1}$ had an atom. This contradiction completes the proof.

\section{NOTE ADDED IN PROOF}

A different direct proof of non-atomicity has been given by Huff; the proof is a Fourier analytic argument based upon results of random walk, whereas the present approach is sample function analytic. See B. W. Huff, Random walks and the continuity of infinitely divisible distributions, SIAM J. Appl. Math. 26 (1974), 372-375.

\section{REFERENCES}

J. R. Blum and M. Rosenblatt, On the structure of infinitely divisible distributions, Pacific J. Math. 9 (1959), 1-7.

R. M. Blumenthal, An extended Markov property, Trans. Amer. Math. Soc. 85 (1957), 52-72.

R. M. Blumenthal and R. K. Getoor, Markov processes and potential theory, Academic Press, New York, 1968.

L. Breiman, Probability, Addison-Wesley, Reading, MA, 1968.

I. I. Gikhman and A. V. Skorokhod, Introduction to the theory of random process, W. B. Saunders, London, 1969.

Philip Hartman and Auriel Wintner, On the infinitesimal generators of integral convolutions, Amer. J. Math. 64 (1942), 273-298.

M. Loève, Probability theory, 4th ed., Springer-Verlag, Berlin and New York, 1977.

Department of Statistics, University of California, Berkeley, California 94720

E-mail address: millarestat.berkeley.edu 